CASE REPORT

T. Katsube

T. Shimono

R. Ashikaga

M. Hosono

H. Kitagaki

T. Murakami

\section{Demonstration of Cerebellar Atrophy in Neuroacanthocytosis of 2 Siblings}

SUMMARY: Neuroacanthocytosis is a rare hereditary disorder characterized by involuntary choreiform movements and erythrocytic acanthocytosis in the peripheral blood. Clinical manifestations of this disorder resemble those of Huntington disease (HD). Neuroimaging features of neuroacanthocytosis are atrophy and signal intensity change of the striata on MR imaging, as in HD. We report herein the cases of 2 siblings with neuroacanthocytosis showing cerebellar atrophy as well as atrophy and signal intensity changes of striata.
$\mathbf{N}$ euroacanthocytosis is a rare, hereditary, heterogeneous, neurodegenerative disorder characterized by adult-onset involuntary choreiform movement and erythrocytic acanthocytosis in the peripheral blood, first described by Citchley et $\mathrm{al}^{1}$ and Levine et al. ${ }^{2}$ Neuroacanthocytosis is mainly classified into autosomal recessive chorea-acanthocytosis (ChAc) and X-linked McLeod syndrome (MLS). The clinical manifestations of neuroacanthocytosis resemble those of Huntington disease (HD), including chorea, cognitive impairment, and psychiatric symptoms.

Neuroimaging features of neuroacanthocytosis are atrophy of the striata, particularly the caudate nucleus, and increased signal intensity from the striata on T2-weighted imaging similar to that seen in HD. ${ }^{3-5}$

To the best of our knowledge, cerebellar atrophy associated with neuroacanthocytosis has been described in only 2 articles. ${ }^{6,7}$ We report herein the cases of 2 siblings with ChAc showing cerebellar atrophy along with atrophy and signal intensity changes in striata on MR imaging.

\section{Case Reports}

\section{Case 1}

A 35-year-old man presented with exacerbation of involuntary movements and a $10-\mathrm{kg}$ weight loss during the course of 1 year. Orofaciolingual dyskinesia had occurred for the past 2.5 years, whereas athetosis, dyskinesia, and chorea of the trunk and extremities had been present for 1.5 years. The parents were second cousins and were alive and without any signs of involuntary movements or neurologic symptoms.

On physical examination, weight was $50 \mathrm{~kg}$ and height was 180 $\mathrm{cm}$. The right eye was congenitally blind because of microphthalmia, and the left eye showed limited light perception.

Neurologic examination revealed orofaciolingual dyskinesia because of autophagia and choreal involuntary movements of the face, trunk, and occasionally the extremities. There was hypotonia and generalized muscle atrophy. Hyporeflexia was present. However, results of the manual muscle test were not decreased. No sensory dis-

Received June 25, 2008; accepted after revision July 3.

From the Department of Radiology (T.K., T.S., R.A., M.H., T.M.), Kinki University School of Medicine, Osaka-Sayama, Japan; and Department of Radiology (H.K.), Shimane University School of Medicine, Izumo City, Shimane-Ken, Japan.

Please address correspondence to Takashi Katsube, MD, Kinki University School of Medicine, 377-2, Ohno-Higashi, Osaka-Sayama, Osaka 589-8511, Japan; e-mail: katsube@radiol.med.kindai.ac.jp

DOI 10.3174/ajnr.A1282 turbance was apparent, and autonomic function was normal. MiniMental State Examination revealed normal mental status.

On laboratory examination, serum creatine kinase level was elevated at $839 \mathrm{IU} / \mathrm{L}$. Serum cholesterol, triglyceride, and $\beta$-lipoprotein levels were normal. Electron microscopy revealed $18 \%$ acanthocytes compared with less than $2 \%$ in healthy control subjects. Kell antigen on erythrocytes was normal.

Results of MR imaging examination of the brain revealed atrophy of the bilateral caudate nuclei and mild signal hyperintensity of the bilateral atrophic putamina on T2-weighted imaging (Fig 1). Cerebral and cerebellar atrophy were also demonstrated.

Genetic screening was declined and was not performed. The patient was diagnosed with $\mathrm{ChAc}$ on the basis of clinical manifestations and findings of brain MR imaging and blood examinations.

\section{Case 2}

A 32-year-old man, the younger brother of the patient in case 1, presented with mild disturbance of talking and ataxia.

He displayed mild oral involuntary movements, muscular hypotonia, and occasional mild choreal involuntary movements. No abnormalities of the eyes were apparent, unlike the patient in case 1 .

MR imaging of the brain showed atrophy of bilateral striata, mildly increased signal intensity of the putamen on T2-weighted imaging, and cerebral atrophy and cerebellar atrophy as in case 1 (Fig 2).

On laboratory examination, serum creatine kinase level was elevated to $1068 \mathrm{IU} / \mathrm{L}$, whereas other levels were normal. ChAc was also diagnosed from clinical manifestations and findings from brain MR imaging and blood examination. Genetic screening was not performed.

\section{Discussion}

The term neuroacanthocytosis is normally used to refer to ChAc and MLS. However, other movement disorders may display erythrocyte acanthocytosis, such as Huntington diseaselike 2 and pantothenate kinase-associated neurodegeneration. ${ }^{8-10}$ Abetalipoproteinemia, which is included under the umbrella term of neuroacanthocytosis, is characterized by the absence of serum apolipoprotein B; symptoms include spinocerebellar ataxia and peripheral neuropathy without involuntary movement. ${ }^{11}$ Abetalipoproteinemia was not considered present in the patients described herein, based on the normal $\beta$-lipoprotein profiles and clinical symptoms.

The characteristic clinical features of ChAc are orofaciolingual dyskinesias, including feeding dystonia and self-mutilation. Conversely, characteristic clinical features of MLS consist of myopathy, hepatosplenomegaly, and heart disease. ${ }^{9,12}$ On laboratory examination, elevated serum creatine kinase levels 

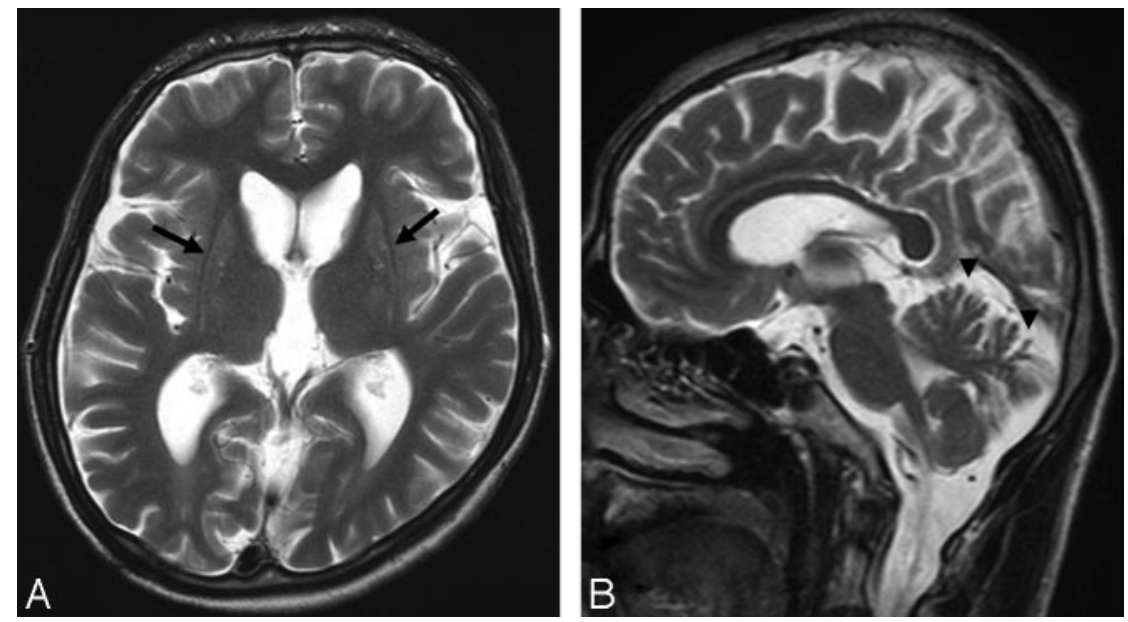

Fig 1. Case 1. T2-weighted MR imaging examination of the brain. A, Axial image showing atrophy of bilateral striata and mildly increased signal intensity from the putamen (arrow). $B$, Sagittal image showing atrophy in the superior cerebellum (arrowhead).
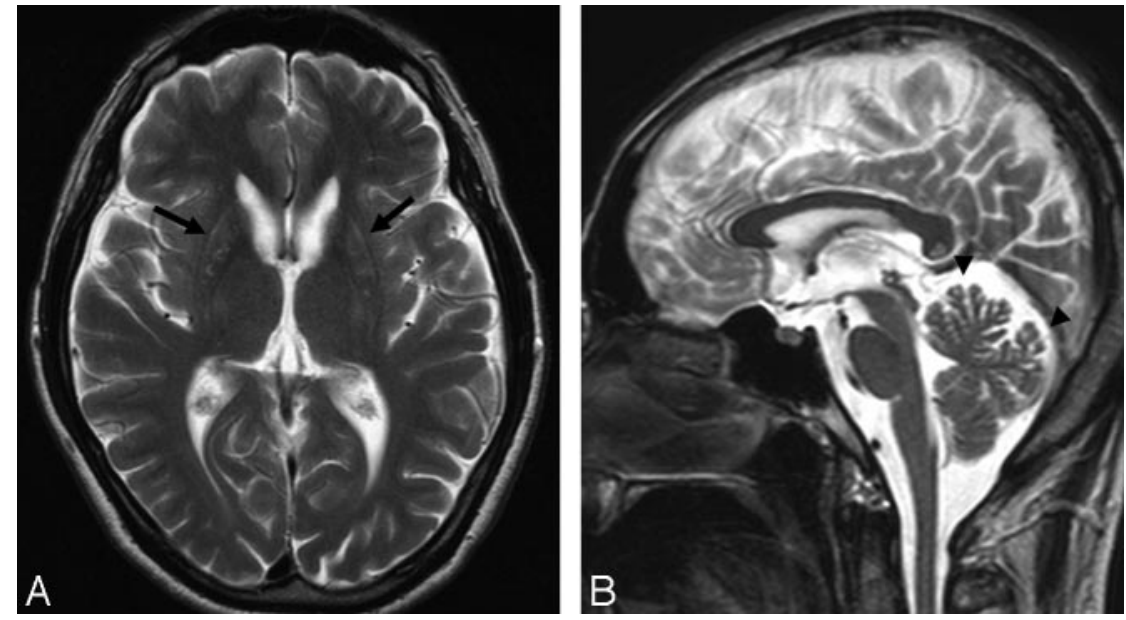

Fig 2. Case 2. T2-weighted MR imaging examination of the brain. Atrophy of bilateral striata, mildly increased signal intensity of the putamen (arrow, A) and cerebellar atrophy (arrowhead, B) are apparent, as in case 1.

and acanthocytosis are observed in both diseases. Absence or weakness of Kell antigen is a characteristic feature of MLS.

Both of the patients in our case report fulfilled the criteria for ChAc. Patient 1 had microphthalmia of the right eye and decreased visual acuity, whereas patient 2 , the younger brother of patient 1 , showed no visual handicaps, and visual handicaps associated with ChAc have not previously been reported. Thus, we consider that associations between the visual handicaps of patient 1 and ChAc may be poor.

The clinical manifestations of neuroacanthocytosis, ChAc, and MLS resemble HD with chorea, cognitive impairment, and psychiatric symptoms. ${ }^{9}$ Characteristic features of neuroacanthocytosis such as orofaciolingual dyskinesia and acanthocytosis help to distinguish this condition from HD. However, overlapping clinical manifestations between neuroacanthocytosis and $\mathrm{HD}$ and the low sensitivity for detection of acanthocytosis on microscopic examination preclude the diagnosis of neuroacanthocytosis. Genetic examination is very useful but is not simple to perform.

On neuroimaging, the characteristic findings of ChAc and MLS resemble those of HD with atrophy of the bilateral caudate nuclei and putamina, with or without increased sig- nal intensity on T2-weighted imaging. ${ }^{3-5}$ Frontal lobe atrophy or general cerebral atrophy has also been reported. ${ }^{4,13,14}$ To the best of our knowledge, cerebellar atrophy in neuroacanthocytosis, as seen in the cases of our 2 patients, has only been mentioned in 2 articles. ${ }^{6,7}$ Tsai et $\mathrm{al}^{6}$ reported 1 case of obvious cerebellar folia atrophy without any abnormality of basal ganglia like spinocerebellar degeneration, though whether the case involved ChAc or MLS was unclear. Nicholl et $\mathrm{al}^{7}$ reported 1 case of cerebellar atrophy and hyperintense periventricular white matter. Although some reports have described cerebellar preservation in patients with neuroacanthocytosis, ${ }^{14,15}$ we consider cerebellar atrophy as an additional finding of neuroacanthocytosis. Cerebellar atrophy on neuroimaging may have been overlooked in cases diagnosed by CT alone without MR imaging because of involuntary movements.

At present, neuroacanthocytosis cannot be distinguished from HD solely on the basis of neuroimaging without clinical manifestations, laboratory examinations, and genetic examinations. In HD, cerebral atrophy is mentioned as a common finding, whereas cerebellar atrophy is mentioned as a minor finding. Rodda ${ }^{16}$ reported only 3 of 300 cases of HD displaying 
severe cerebellar atrophy. Cerebellar atrophy may thus help to distinguish neuroacanthocytosis from HD on neuroimaging.

In conclusion, we have reported herein the cases of 2 siblings showing cerebellar atrophy along with striatal atrophy and signal intensity changes of the putamina. In addition to atrophy and striatal signal intensity changes mentioned as a characteristic finding of neuroacanthocytosis on neuroimaging, cerebellar atrophy may represent an additional finding for neuroacanthocytosis. More images of patients with neuroacanthocytosis should be accumulated to confirm the presence of cerebellar atrophy, with the hope that this manifestation will enable differentiation between neuroacanthocytosis and HD on neuroimaging.

\section{References}

1. Citchley EM, Clark DB, Wikler A. Acanthocytosis and neurological disorder without abetalipoproteinemia. Arch Neurol 1968;18:134-40

2. Levine IM, Estes JW, Looney JM. Hereditary neurological disease with acanthocytosis: a new syndrome. Arch Neurol 1968;19:403-09

3. Sundaram SM, Kumar MJ, Sridhar R, et al. Neuroacanthocytosis misdiagnosed as Huntington's disease: a case report. J Neurol Sci 2004;219:163-66
4. Kutcher JS, Kahn MJ, Andersson HC, et al. Neuroacanthocytosis masquerading as Huntington's disease: CT/MRI findings. J Neuroimaging 1999;9:187-89

5. Okamoto K, Ito J, Furusawa T, et al. CT and MR findings of neuroacanthocytosis. J Comput Assist Tomogr 1997;21:221-22

6. Tsai $\mathrm{CH}$, Chen RS, Chang HC, et al. Acanthocytosis and spinocerebellar degeneration: a new association? Mov Disord 1997;12:456-59

7. Nicholl DJ, Sutton I, Dotti MT, et al. White matter abnormalities on MRI in neuroacanthocytosis. J Neurol Neurosurg Psychiatry 2004;75:1200-01

8. Walker RH, Jung HH, Dobson-Stone C, et al. Neurologic phenotypes associated with acanthocytosis. Neurology 2007;68:92-98

9. Danek A, Walker RH. Neuroacanthocytosis. Curr Opin Neurol 2005;18:386-92

10. Danek A, Jung HH, Melone MA, et al. Neuroacanthocytosis: new developments in a neglected group of dementing disorders. J Neurol Sci 2005;229-230:171-86

11. Rampoldi L, Danek A, Monaco AP. Clinical features and molecular bases of neuroacanthocytosis. J Mol Med 2002;80:475-91

12. Sakai T, Mawatari S, Imashita H, et al. Choreoacanthocytosis: clues to clinical diagnosis. Arch Neurol 1981;38:335-38

13. Bohlega S, Al-Jishi A, Dobson-Stone C, et al. Chorea-acanthocytosis: clinical and genetic findings in three families from the Arabian peninsula. Mov Disord 2003;18:403-07

14. Hardie RJ, Pullon HW, Harding AE, et al. Neuroacanthocytosis. A clinical, haematological and pathological study of 19 cases. Brain 1991;114:13-49

15. Rinne JO, Daniel SE, Scaravilli F, et al. The neuropathological features of neuroacanthocytosis. Mov Disord 1994;9:297-304

16. Rodda RA. Cerebellar atrophy in Huntington's disease. J Neurol Sci 1981;50: $147-57$ 